\title{
Objective response of first-line chemotherapy of triple-negative breast cancer translates into survival benefit: an analysis in an independent, prospective clinical trial and a real-world setting
}

\author{
T. LI ${ }^{1,2,4}$, Y. H. ZHU ${ }^{1,2, *}$, J. ZHANG ${ }^{1,2, *}$, B. Y. WANG ${ }^{1,2}$, S. ZHANG ${ }^{1,2}$, L. P. WANG ${ }^{1,2}$, J. CAO ${ }^{1,2}$, Z. H. TAO ${ }^{1,2}$, J. XIE ${ }^{1,2}$, X. C. HU ${ }^{1,2, *}$ \\ ${ }^{1}$ Department of Medical Oncology, Shanghai Cancer Center, Fudan University, Shanghai, China; ${ }^{2}$ Department of Oncology, Shanghai Medical \\ College, Fudan University, Shanghai, China
}

*Correspondence: huxichun2019@163.com

${ }^{*}$ Contributed equally to this work.

Received February 25, 2020/ Accepted March 30, 2020

\begin{abstract}
This study sought to assess whether the objective response (OR, including complete response and partial response) of first-line chemotherapy can predict overall survival (OS) for patients with metastatic triple-negative breast cancer (mTNBC) in both clinical trial and a real-world setting. The survival predictable parameters were assessed in two independent cohorts, the training cohort of 236 patients as part of a phase 3 trial (CBCSG006, Trial registration number NCT0128762) and the validation cohort of 360 patients from the real-world setting. Univariable and multivariable Cox proportional hazard models were applied to explore associations with progression-free survival and OS in the training cohort and then in the validation cohort. OR (OR vs non-OR, HR, 0.438, p<0.001) together with Eastern Cooperative Oncology Group (ECOG) performance status, disease-free survival, number of metastatic organ sites and platinum-based chemotherapy used as firstline chemotherapy were observed to be independent prognostic factors for progression-free survival (PFS), and OR (OR vs non-OR, HR, 0.602, $\mathrm{p}=0.002$ ) together with ECOG score, disease-free survival, number of metastatic organ sites and previous anthracycline and/or taxane treatment were observed to be independent predictive factors for OS in the training cohort. These predictors were confirmed in the validation cohort. For OR and non-OR group, median OS was 23.72 and 13.83 months in the training cohort (HR, 0.637, $\mathrm{p}=0.002)$, and 21.95 and 13.80 months in the validation cohort (HR, 0.608, $\mathrm{p}<0.001$ ), respectively. By adding OR in the OS predictors, the concordance index (C-index) improved from 0.622 to 0.645 in the training cohort and 0.653 to 0.675 in the validation cohort. PFS and OS of MTNBC can be predicted by OR status with any regimen of first-line chemotherapy in an independent prospective clinical trial and a real-world setting. Therefore, TNBC, not like other subtypes of breast cancer, may be in need of combination chemotherapy or intense chemotherapy to achieve a high response rate for survival.
\end{abstract}

Key words: first-line chemotherapy, metastatic triple-negative breast cancer, objective response, overall survival

Triple-negative breast cancer (TNBC) is a special subtype of breast cancer with poor prognosis. It is defined as the absence of estrogen receptor (ER) and progesterone receptor (PR) as well as human epidermal growth factor receptor 2 (HER-2) amplification. TNBC comprises about $15 \%$ of all invasive breast cancers and is characterized by larger tumors, higher-grade tumors, more rapid growth of tumors, and more frequent metastasis to viscera such as lungs and brain. Patients with TNBC are not eligible to be treated with endocrine therapies or anti-HER2 targeted therapies [1]. For patients with a germline breast cancer susceptibility gene (BRCA) mutation, Olaparib monotherapy provided an option. However, the mutation rate was low, and no overall survival (OS) prolongation was achieved [2]. Therefore, cytotoxic chemotherapy currently remains the main systemic therapy. Patients with TNBC attain pathologic complete response (pCR) rates of $30-40 \%$ in the neoadjuvant setting, which is known as a predictor for long-term survival [3]. Despite the significant impact of pCR rates on outcomes, no standard and, more importantly, effective therapy exists for this population when metastasis occurs. A vast majority of deaths occur in the first 5 years after treatment, indicating that TNBC, as a whole group, still has a poor outcome [1].

Patients with continued complete response (CR) following the first-line treatment had longer survival [4], and the longer duration of first-line chemotherapy was associated 
with prolonged OS and progression-free survival (PFS) [5]. However, objective response (OR) predicting survival in metastatic triple-negative breast cancer (mTNBC) in a firstline setting has not been well studied. Therefore, a better understanding of the relationship between response and survival in this setting is urgently required.

This study investigated the clinical characteristics of patients in both clinical trial and a real-world setting with the intention to determine the OR (including CR and PR) in the risk prognostication of OS for patients with metastatic TNBC (mTNBC) receiving first-line chemotherapy. The results might provide a strategy for choosing combination chemotherapy or sequential single-agent chemotherapy in the first-line treatment of mTNBC.

\section{Patients and methods}

Patient selection. The risk prognostic parameters were assessed in two independent cohorts of patients with mTNBC receiving first-line chemotherapy. The training cohort was derived from the randomized CBCSG006 trial in Fudan University Shanghai Cancer Center, recruiting 236 patients between January 2011 and November 2013. The flow chart was described as previously and its_primary results had already been published [6]. The outcome data of the original CBCSG006 trial were updated in this study. The validation cohort was from a breast cancer population of 360 patients with mTNBC recruited between April 2005 and November 2013 in the real-world setting. All data were collected from electronic patient records and hospital charts. The flow chart of the validation cohort was shown in Figure 1. The study procedures were approved by the institutional ethical board of the Fudan University Shanghai Cancer Center. The eligible patients were $\geq 18$ years old, had TNBC histologically confirmed as the primary tumor, and had clinical, imaging, histological, or cytological evidence of metastatic disease. Patients were classified as TNBC based on their surgical or biopsy results. ER and $\mathrm{PR}$ were considered negative if less than $1 \%$ of tumor cells exhibited nuclear staining, and HER2 negative was defined as having an immunohistochemistry (IHC) score of 0 or $1+$ or fluorescence in situ hybridization (FISH) non-amplified score according to the American Society of Clinical Oncology (ASCO) guidelines [7, 8]. Patients with an incomplete receptor status or a changed status in metastatic lesions inconsistent with the aforementioned definition were excluded.

Data collection. The efficacy was analyzed according to the overall response rate (ORR), PFS, and OS. Tumor response was evaluated in accordance with the Response Evaluation Criteria in Solid Tumors (RECIST 1.1) guidelines by computed tomography scanning or magnetic resonance imaging every two cycles or as clinically indicated [9]. The date of disease progression was determined from the clinical notes. OR was defined as CR and partial response (PR) using RECIST, version 1.1. The ORR was defined as the percentage of patients who achieved a CR and a PR using RECIST, version 1.1. PFS was defined as the time from the start of the first-line treatment until disease progression or death by any cause. OS was calculated from the start of the first-line treatment to death by any cause or the last date when the patient was

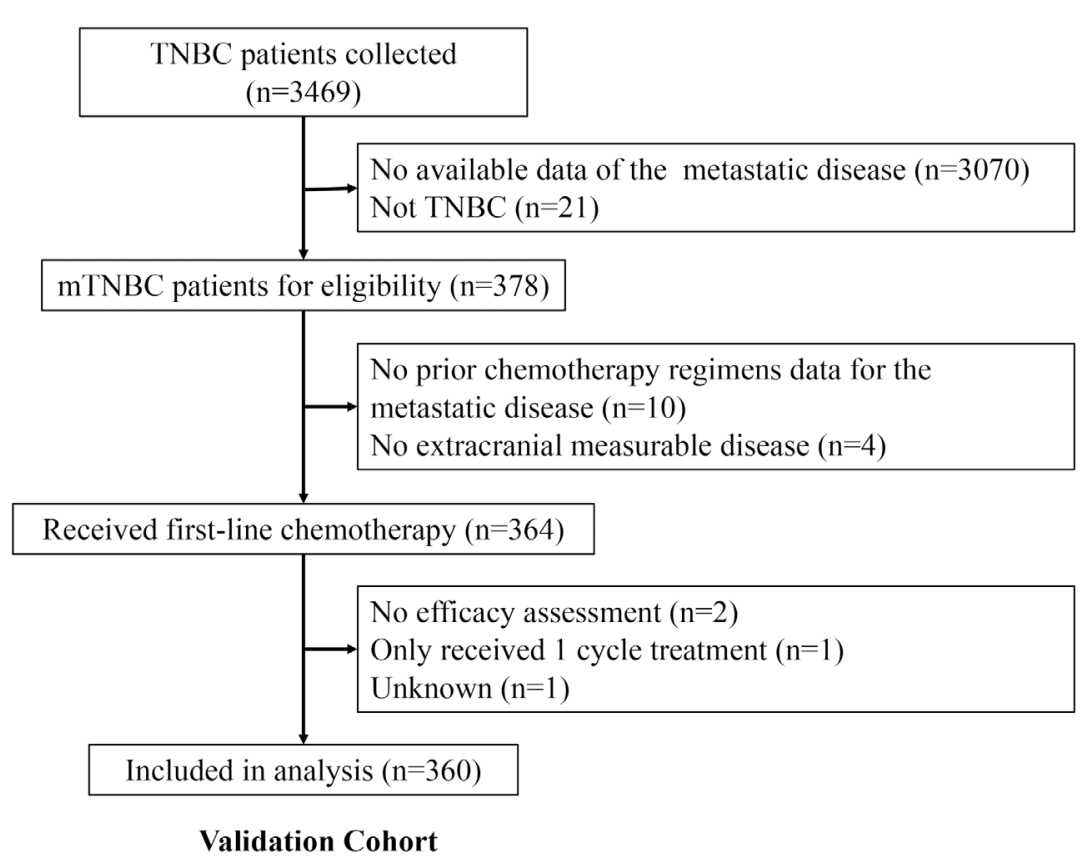

Figure 1. Flow chart of 360 enrolled patients with mTNBC. TNBC, triple-negative breast cancer; mTNBC, metastatic TNBC. 
known to be alive. Potential prognostic variables, including age, Eastern Cooperative Oncology Group (ECOG) performance status, menstruation status, details about neoadjuvant and adjuvant chemotherapy, disease-free interval (DFI), number and sites of metastatic disease, and OR status, were collected at the time of diagnosis of distant metastases or the beginning of treatment.

Statistical analysis. The risk prognostic parameters were assessed in two independent cohorts. First, univariate and multivariate Cox proportional hazards models were applied to explore associations with PFS and OS in the training cohort. Then, the prognostic parameters were validated in the validation cohort. The results were analyzed using SPSS 16.0 (SPSS, IL, USA). The statistical analysis of $2 \times 2$ contingency tables of categorical variables in the two cohorts was performed using the Pearson's chi-square test. Factors with $\mathrm{p}<0.1$ in the univariate analysis were examined with the Cox proportional hazards model, which defined independent predictive factors, hazard ratios (HRs), and 95\% CIs. The median PFS and OS were estimated by the Kaplan-Meier method and compared using a log-rank test. All p-values were two sided, and a p-value $<0.05$ was considered to indicate a statistically significant difference. Additionally, the C-index (Harrell) was calculated for prognostic discrimination using the statistical software $\mathrm{R}$ (version 3.1.0) and the R-package rms (version 1.0.1).

\section{Results}

Patients' characteristics. The characteristics of the study participants are presented in Table 1 . The training and validation cohorts comprised of 236 and 360 patients, respectively. No significant difference was found in the distribution of age, menstruation status, number of metastatic sites, and anthracyclines and taxanes in prior neoadjuvant/adjuvant chemotherapy between the two cohorts. In the training cohort, the participants had a better ECOG performance status $(\mathrm{p}=0.003)$ and a longer DFI $(\mathrm{p}=0.001)$ compared with the validation cohort. Further, more patients had visceral metastases in the training cohort $(70.8 \%$ vs. $57.2 \%, \mathrm{p}=0.001)$. The metastatic sites were well balanced between the two cohorts except for the metastatic site in the lung ( $56.8 \%$ vs. $44.7 \%, \mathrm{p}=0.004$ ). Fewer patients used platinum-based chemotherapy (PBCT) as first-line chemotherapy in the training cohort compared with the validation cohort ( $50.0 \%$ vs. $60.0 \%, \mathrm{p}=0.016$ ).

Training cohort: survival and predictors of survival. By the time of data lock, the median PFS was 6.54 months (95\% CI 5.83-7.25 months) and the median OS was 18.86 months (95\% CI 15.14-22.58 months), with the ORR of 56.8\% (12 $\mathrm{CR}$ and $122 \mathrm{PR}$ ) for the training cohort (Figures 2A, 2B). The results of the univariate analysis are listed in Table 2. OR $(\mathrm{p}<0.001$, Figure $2 \mathrm{C})$ together with DFI $(\mathrm{p}=0.015)$, number of metastatic organ sites $(\mathrm{p}<0.001)$, and PBCT used as firstline chemotherapy $(p=0.003$, Figure $2 \mathrm{D}$ ) were observed to be predictive factors for better PFS. OR $(\mathrm{p}=0.002$, Figure $2 \mathrm{E})$
Table 1. Characteristics of study participants in the training and validation cohorts.

\begin{tabular}{|c|c|c|c|c|c|}
\hline \multirow[t]{2}{*}{ Characteristics } & \multicolumn{2}{|c|}{$\begin{array}{l}\text { Training } \\
(\mathbf{n}=\mathbf{2 3 6})\end{array}$} & \multicolumn{2}{|c|}{$\begin{array}{c}\text { Validation } \\
(\mathrm{n}=360)\end{array}$} & \multirow[t]{2}{*}{ p-value } \\
\hline & $\mathbf{n}$ & $\%$ & $\mathbf{n}$ & $\%$ & \\
\hline Age (median, range), years & \multicolumn{2}{|c|}{$47,26-72$} & \multicolumn{2}{|c|}{$49,25-76$} & \\
\hline$<40$ & 40 & 16.9 & 72 & 20.0 & 0.351 \\
\hline$\geq 40$ & 196 & 83.1 & 288 & 80.0 & \\
\hline \multicolumn{6}{|l|}{ Menstruation status } \\
\hline Postmenopausal & 117 & 49.6 & 186 & 51.7 & 0.618 \\
\hline Premenopausal & 119 & 50.4 & 174 & 48.3 & \\
\hline \multicolumn{6}{|l|}{ ECOG performance status } \\
\hline 0 & 81 & 34.3 & 84 & 23.3 & 0.003 \\
\hline$\geq 1$ & 155 & 65.7 & 276 & 76.7 & \\
\hline \multicolumn{6}{|l|}{ Number of metastatic sites } \\
\hline$<3$ & 152 & 64.4 & 253 & 70.3 & 0.133 \\
\hline$\geq 3$ & 84 & 35.6 & 107 & 29.7 & \\
\hline \multicolumn{6}{|l|}{ Metastatic sites } \\
\hline Lymph nodes & 144 & 61.0 & 224 & 62.2 & 0.767 \\
\hline Liver & 60 & 25.4 & 78 & 21.7 & 0.288 \\
\hline Bone & 56 & 23.7 & 98 & 27.2 & 0.341 \\
\hline Lung & 134 & 56.8 & 161 & 44.7 & 0.004 \\
\hline Chest wall & 52 & 22.0 & 100 & 27.8 & 0.116 \\
\hline Pleura & 22 & 9.3 & 32 & 8.9 & 0.857 \\
\hline Brain & 10 & 4.2 & 11 & 3.1 & 0.444 \\
\hline Contralateral breast & 10 & 4.2 & 7 & 1.9 & 0.100 \\
\hline \multicolumn{6}{|l|}{ Visceral metastasis } \\
\hline Yes & 167 & 70.8 & 206 & 57.2 & 0.001 \\
\hline No & 69 & 29.2 & 154 & 42.8 & \\
\hline \multicolumn{6}{|l|}{ Disease-free interval, month } \\
\hline$>12$ & 171 & 72.5 & 205 & 56.9 & 0.001 \\
\hline$\leq 12$ & 65 & 27.5 & 155 & 43.1 & \\
\hline \multicolumn{6}{|l|}{ Prior adjuvant chemotherapy } \\
\hline Anthracyclines & 195 & 82.6 & 286 & 79.4 & 0.336 \\
\hline Taxanes & 152 & 64.4 & 207 & 57.5 & 0.092 \\
\hline Both & 139 & 58.9 & 183 & 50.8 & 0.053 \\
\hline \multicolumn{6}{|c|}{ PBCT used as first-line chemotherapy } \\
\hline Yes & 118 & 50.0 & 216 & 60.0 & 0.016 \\
\hline No & 118 & 50.0 & 144 & 40.0 & \\
\hline
\end{tabular}

Abbreviations: ECOG - Eastern Cooperative Oncology Group; PBCT - platinum-based chemotherapy

together with DFI $(\mathrm{p}<0.001)$ and number of metastatic organ sites $(p<0.001)$ were observed to be predictive factors for better OS. For OR and non-OR groups, the median OS was 23.72 and 13.83 months in 134 and 102 patients, respectively (HR, $0.637, p=0.002$, Figure $2 \mathrm{E}$ ). No difference was found in the OS between the PBCT and non-PBCT groups (Figure 2F).

All significant factors in the univariate analysis were included in the multivariate analysis based on Cox regression. OR (OR vs. non-OR, HR, 0.438, 95\% CI 0.324-0.592, $\mathrm{p}<0.001)$ together with the ECOG performance status $(p=0.001)$, DFI $(p=0.023)$, number of metastatic organ 

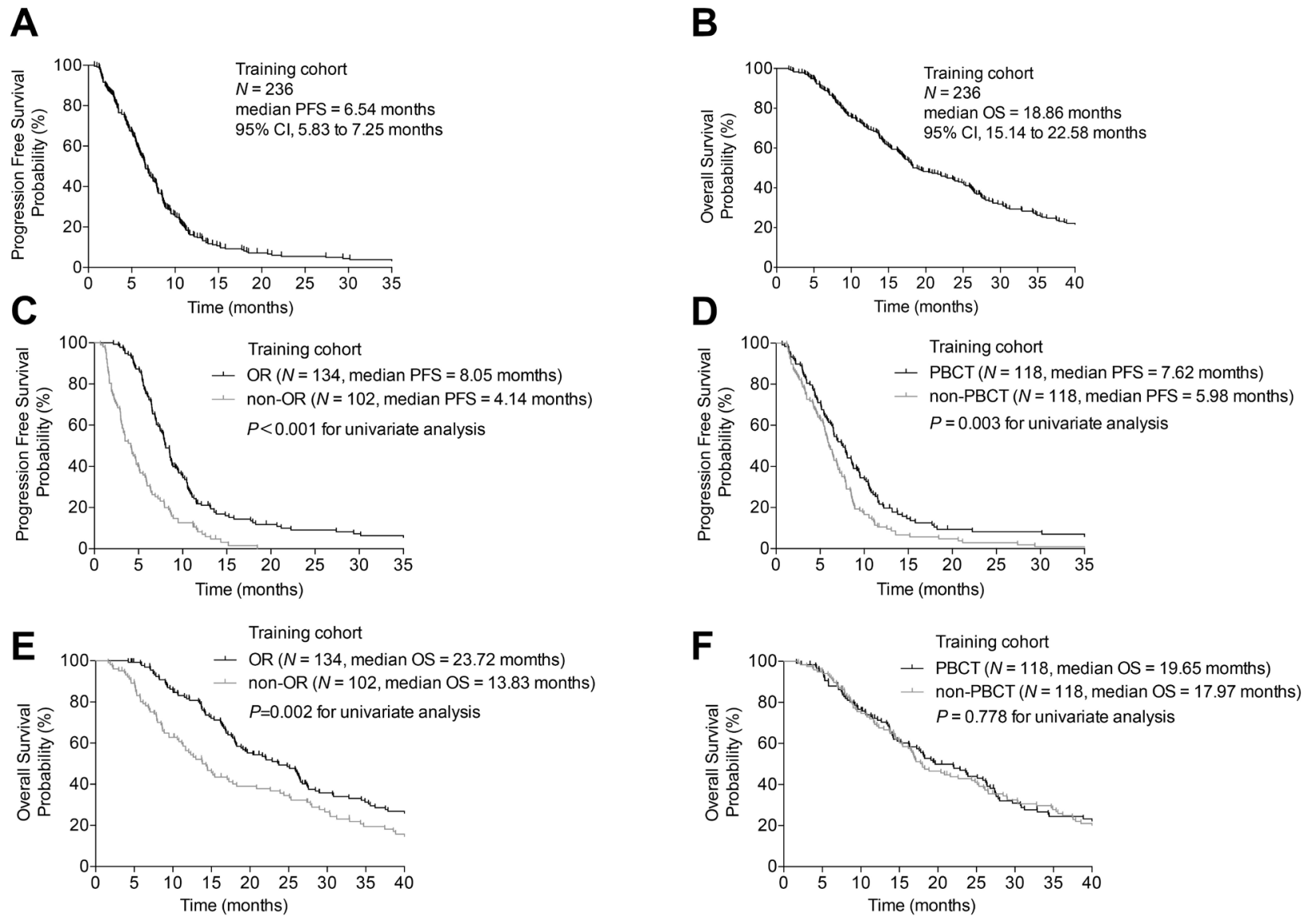

Figure 2. Survival and predictors of survival in the training cohort: Kaplan-Meier plot of PFS (A) and OS (B) for all patients; Kaplan-Meier plot of PFS (C) and OS (E) in patients with OR versus non-OR; Kaplan-Meier plot of PFS (D) and OS (F) in patients with PBCT versus non-PBCT.

sites $(\mathrm{p}<0.001)$, and PBCT used as first-line chemotherapy $(\mathrm{p}=0.004)$ remained independent prognostic factors for PFS. OR (OR vs. non-OR, HR, 0.602, 95\% CI 0.440-0.824, $\mathrm{p}=0.002)$ together with ECOG $(\mathrm{p}=0.029)$, DFI $(\mathrm{p}<0.001)$, number of metastatic organ sites $(\mathrm{p}<0.001)$, and previous treatment (anthracycline and/or taxane) $\quad(\mathrm{p}=0.022)$ remained independent prognostic factors for OS (Table 3). The predictive accuracy and discriminative ability were measured using the concordance index (C-index). By adding OR in the OS predictors, including age, menstruation status, ECOG status, DFI, number of metastatic sites, visceral metastasis, $\mathrm{PBCT}$, and previous treatment (anthracycline and/or taxane), the C-index improved from 0.622 to 0.645 in the training cohort.

Validation cohort: survival and predictors of survival. By the time of data lock, the median PFS was 6.54 months (95\% CI 5.80-7.28 months) and the median OS was 17.05 months (95\% CI 15.23-18.87 months), with the ORR of $47.8 \%$ (23 CR and $149 \mathrm{PR}$ ) for the validation cohort (Figures 3A, 3B). The parameters estimated from the training cohort were verified in the independent valida- tion cohort. The results of univariate analyses are listed in Table 2. In the univariate analyses, longer PFS was observed in patients with ECOG performance status for $0(\mathrm{p}=0.005)$, DFI $\geq 12(p=0.012)$, number of metastatic organ sites $<3$ $(\mathrm{p}<0.001)$, OR $(\mathrm{p}<0.001$, Figure $3 \mathrm{C})$, and PBCT used as first-line chemotherapy $(p<0.001$, Figure $3 D)$. Longer OS was observed in patients with postmenopausal status $(p=0.026)$, DFI $(p<0.001)$, number of metastatic organ sites $<3(\mathrm{p}<0.001)$, no visceral metastasis $(\mathrm{p}=0.036)$, and $\mathrm{OR}(\mathrm{p}<0.001$, Figure $3 \mathrm{E})$. For the OR and non-OR groups, the median OS was 21.95 and 13.80 months in 172 and 188 patients, respectively (HR, $0.608, \mathrm{p}<0.001$, Figure $3 \mathrm{E}$ ). The results were consistent with those from the training cohort. No difference in OS was observed between the PBCT and non-PBCT groups (Figure 3F).

A Cox proportional hazards model was used to determine the independent predictive factors of the aforementioned variables of survival for patients with $\mathrm{mTNBC}$ in the first-line setting. OR (OR vs. non-OR, HR, 0.448, 95\% CI $0.353-0.568, \mathrm{p}<0.001)$ together with ECOG score $(\mathrm{p}=0.024)$, DFI $(p=0.021)$, number of metastatic organ sites $(p=0.002)$, 
Table 2. Univariate analyses results of study participants in the training and validation cohorts.

\begin{tabular}{|c|c|c|c|c|c|c|}
\hline \multicolumn{7}{|c|}{ Univariate analysis results of PFS } \\
\hline \multirow{2}{*}{ Variables } & \multicolumn{3}{|c|}{ Training $(\mathrm{n}=236)$} & \multicolumn{3}{|c|}{ Validation $(n=360)$} \\
\hline & Hazard ratio & $95 \% \mathrm{CI}$ & p-value & Hazard ratio & 95\% CI & p-value \\
\hline \multicolumn{7}{|l|}{ Age, years } \\
\hline$<40$ & 1.528 & $0.757-1.528$ & 0.683 & 1.089 & $0.833-1.424$ & 0.533 \\
\hline$\geq 40$ & Reference & & & Reference & & \\
\hline \multicolumn{7}{|l|}{ ECOG } \\
\hline 0 & 0.793 & $0.599-1.050$ & 0.106 & 0.685 & $0.526-0.893$ & 0.005 \\
\hline$\geq 1$ & Reference & & & Reference & & \\
\hline \multicolumn{7}{|l|}{ Menopausal status } \\
\hline Premenopausal & 1.223 & $0.936-1.598$ & 0.140 & 1.199 & $0.968-1.486$ & 0.097 \\
\hline Postmenopausal & Reference & & & Reference & & \\
\hline \multicolumn{7}{|l|}{ DFI } \\
\hline$<12$ & 1.515 & $1.084-2.119$ & 0.015 & 1.339 & $1.066-1.683$ & 0.012 \\
\hline$\geq 12$ & Reference & & & Reference & & \\
\hline \multicolumn{7}{|c|}{ Number of metastatic sites } \\
\hline$<3$ & 0.517 & $0.390-0.686$ & $<0.001$ & 0.614 & $0.484-0.779$ & $<0.001$ \\
\hline$\geq 3$ & Reference & & & Reference & & \\
\hline \multicolumn{7}{|l|}{ Visceral metastasis } \\
\hline No & 0.820 & $0.608-1.105$ & 0.191 & 0.805 & $0.647-1.002$ & 0.053 \\
\hline Yes & Reference & & & Reference & & \\
\hline \multicolumn{7}{|c|}{ Anthracycline and/or taxane } \\
\hline No & 1.030 & $0.785-1.351$ & 0.833 & 0.977 & $0.715-1.335$ & 0.884 \\
\hline Yes & Reference & & & Reference & & \\
\hline \multicolumn{7}{|c|}{$\mathrm{PBCT}$ used as first-line chemotherapy } \\
\hline No & 1.498 & $1.145-1.959$ & 0.003 & 1.544 & $1.240-1.921$ & $<0.001$ \\
\hline Yes & Reference & & & Reference & & \\
\hline \multicolumn{7}{|c|}{ Objective response status } \\
\hline OR & 0.406 & $0.308-0.535$ & $<0.001$ & 0.447 & $0.360-0.555$ & $<0.001$ \\
\hline Non-OR & Reference & & & Reference & & \\
\hline \multicolumn{7}{|c|}{ Univariate analysis results of OS } \\
\hline \multirow{2}{*}{ Variables } & & $g(n=236)$ & & & tion $(n=360$ & \\
\hline & Hazard ratio & $95 \% \mathrm{CI}$ & p-value & Hazard ratio & $95 \% \mathrm{CI}$ & p-value \\
\hline \multicolumn{7}{|l|}{ Age, years } \\
\hline$<40$ & 0.929 & $0.625-1.381$ & 0.715 & 1.087 & $0.823-1.434$ & 0.558 \\
\hline$\geq 40$ & Reference & & & Reference & & \\
\hline ECOG & & & & & & \\
\hline 0 & 0.922 & $0.684-1.244$ & 0.596 & 0.874 & $0.674-1.132$ & 0.307 \\
\hline$\geq 1$ & Reference & & & Reference & & \\
\hline Menopausal status & & & & & & \\
\hline Premenopausal & 1.231 & $0.924-1.640$ & 0.156 & 1.284 & $1.031-1.600$ & 0.026 \\
\hline Postmenopausal & Reference & & & Reference & & \\
\hline DFI & & & & & & \\
\hline$<12$ & 1.950 & $1.365-2.785$ & $<0.001$ & 1.836 & $1.449-2.326$ & $<0.001$ \\
\hline$\geq 12$ & Reference & & & Reference & & \\
\hline Number of metastat & & & & & & \\
\hline$<3$ & 0.466 & $0.345-0.630$ & $<0.001$ & 0.495 & $0.390-0.628$ & $<0.001$ \\
\hline$\geq 3$ & Reference & & & Reference & & \\
\hline Visceral metastasis & & & & & & \\
\hline No & 0.743 & $0.536-1.030$ & 0.074 & 0.787 & $0.630-0.984$ & 0.036 \\
\hline Yes & Reference & & & Reference & & \\
\hline Anthracycline and/o & & & & & & \\
\hline No & 0.830 & $0.617-1.118$ & 0.220 & 0.751 & $0.540-1.043$ & 0.087 \\
\hline Yes & Reference & & & Reference & & \\
\hline PBCT used as first-li & & & & & & \\
\hline No & 1.042 & $0.782-1.387$ & 0.778 & 0.906 & $0.723-1.134$ & 0.387 \\
\hline Yes & Reference & & & Reference & & \\
\hline Objective response s & & & & & & \\
\hline OR & 0.637 & $0.477-0.851$ & 0.002 & 0.608 & $0.488-0.759$ & $<0.001$ \\
\hline Non-OR & Reference & & & Reference & & \\
\hline
\end{tabular}

Abbreviations: DFI - disease-free interval; ECOG - Eastern Cooperative Oncology Group; HR - hazard ratio; MBC - metastatic breast cancer; OR - objective response; OS - overall survival; PBCT - platinum-based chemotherapy; PFS - progression-free survival 

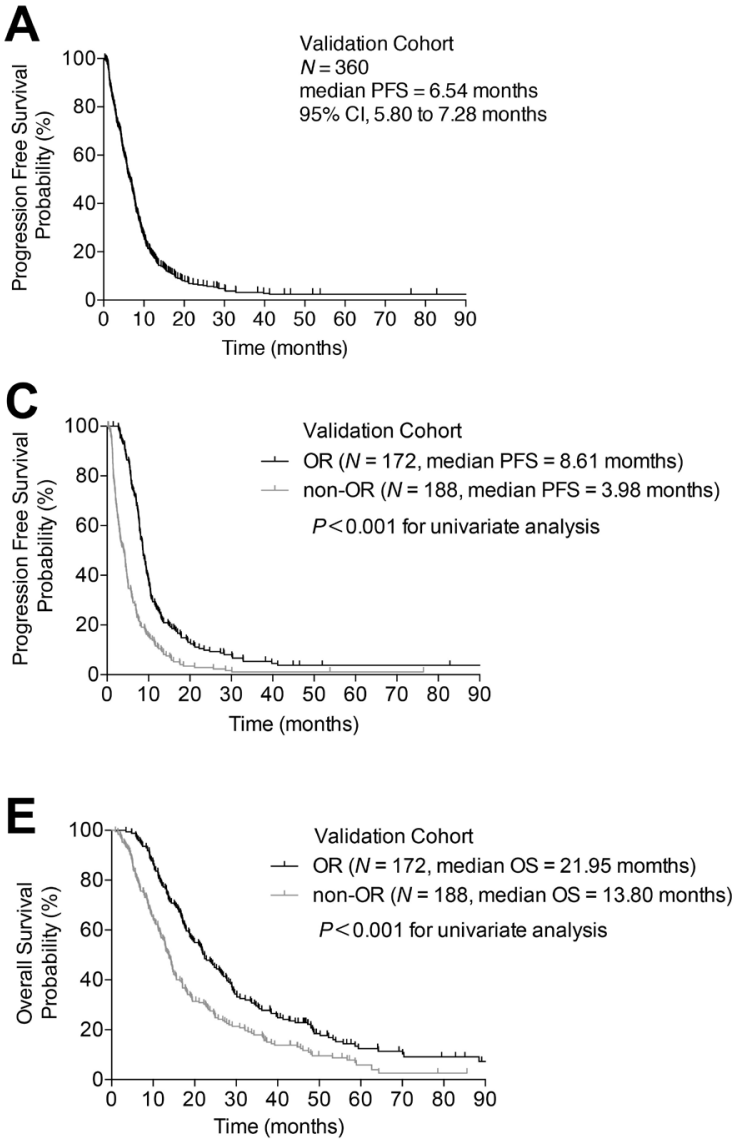

B
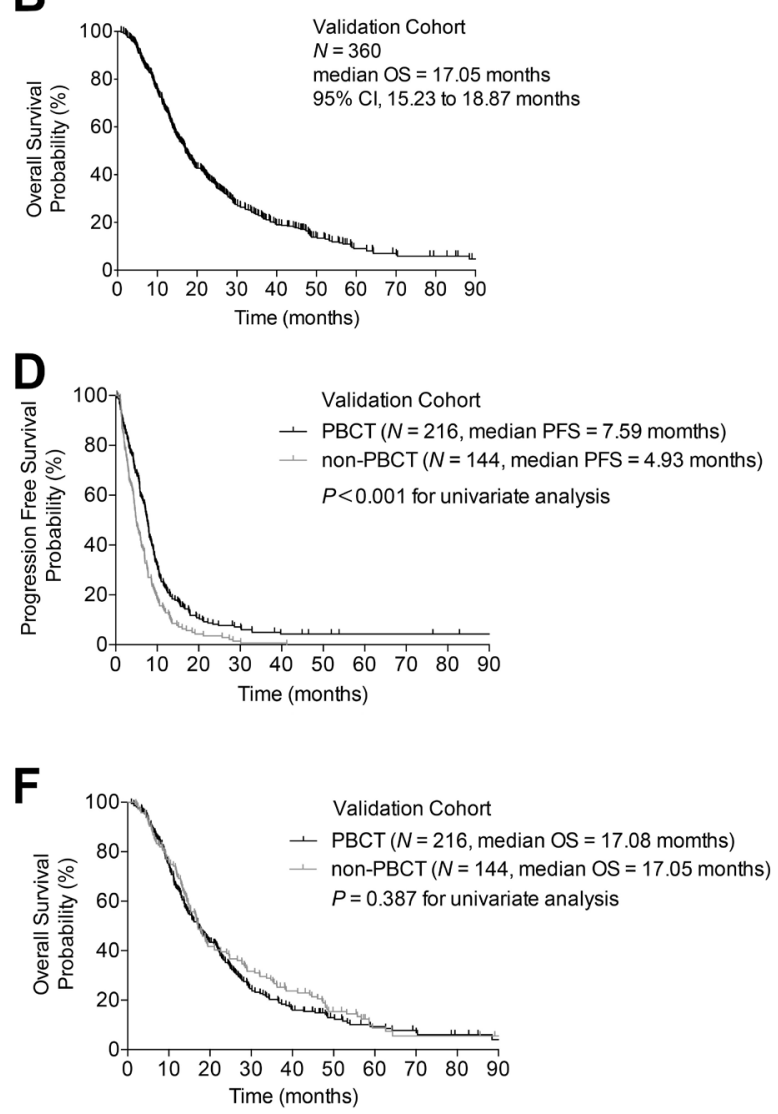

Figure 3. Survival and predictors of survival in the validation cohort: Kaplan-Meier plot of PFS (A) and OS (B) for all patients; Kaplan-Meier plot of PFS (C) and OS (E) in patients with OR versus non-OR; Kaplan-Meier plot of PFS (D) and OS (F) in patients with PBCT versus non-PBCT.

menopausal status ( $\mathrm{p}=0.010)$, and $\mathrm{PBCT}$ used as first-line chemotherapy ( $\mathrm{p}=0.008)$ were observed to be independent predictive factors for PFS. OR (OR vs. non-OR, HR, 0.561, $95 \%$ CI $0.440-0.715, \mathrm{p}<0.001)$ together with DFI $(\mathrm{p}<0.001)$, number of metastatic organ sites $(\mathrm{p}<0.001)$, and previous treatment (anthracycline and/or taxanes; $\mathrm{p}=0.027$ ) were observed to be independent predictive factors for OS. The C-index improved from 0.653 to 0.675 by adding OR in the OS predictors, including age, menstruation status, ECOG status, DFI, number of metastatic sites, visceral metastasis, PBCT, and previous treatment (anthracycline and/or taxane).

\section{Discussion}

TNBC is aggressive breast cancer with poor prognosis. No benefit from endocrine therapy or trastuzumab and the lack of a preferred standard form of chemotherapy has made the treatment of patients with TNBC more difficult, especially when recurrence or metastasis occurs $[10,11]$. Improvement in survival becomes a critical issue. The present study revealed that the first-line OR status with any regimen could predict the PFS and OS of patients with mTNBC in an independent prospective clinical trial and a real-world setting.

pCR was proposed as a surrogate for survival in early TNBC. Compared with other subtypes of breast cancer, patients with TNBC showed high pCR with neoadjuvant treatment, but those who did not achieve pCR had a six-tonine times higher risk for relapse [3, 12]. Recently, increasing pCR in TNBC has been a major focus. Adding either carboplatin or bevacizumab to neoadjuvant chemotherapy increased pCR rates in patients with stage II-III TNBC [13, 14]. Although patients with pCR had improved survival, especially in aggressive tumor subtypes, such as TNBC, the validation of $\mathrm{pCR}$ as a surrogate endpoint for improved eventfree survival and OS was not proved $[15,16]$. In renewed data, the GeparSixto study reported that the addition of carboplatin to a neoadjuvant regimen significantly increased not only the proportion of patients achieving $\mathrm{pCR}$, but also the DFI [17].

The combination chemotherapy showed a statistically significant advantage for survival compared with the sequen- 
Table 3. Cox regression results of study participants in the training and validation cohorts.

\begin{tabular}{|c|c|c|c|c|c|c|}
\hline \multicolumn{7}{|c|}{ Cox regression results of PFS } \\
\hline \multirow{2}{*}{ Variables } & \multicolumn{3}{|c|}{ Training $(\mathrm{n}=236)$} & \multicolumn{3}{|c|}{ Validation $(n=360)$} \\
\hline & Hazard ratio & $95 \% \mathrm{CI}$ & p-value & Hazard ratio & $95 \% \mathrm{CI}$ & p-value \\
\hline \multicolumn{7}{|l|}{ Age, years } \\
\hline$<40$ & 0.989 & $0.651-1.503$ & 0.960 & 1.033 & $0.747-1.430$ & 0.843 \\
\hline$\geq 40$ & Reference & & & Reference & & \\
\hline \multicolumn{7}{|l|}{ ECOG } \\
\hline 0 & 0.594 & $0.433-0.815$ & 0.001 & 0.721 & $0.543-0.958$ & 0.024 \\
\hline$\geq 1$ & Reference & & & Reference & & \\
\hline \multicolumn{7}{|l|}{ DFI } \\
\hline$<12$ & 1.520 & $1.060-2.179$ & 0.023 & 1.319 & $1.043-1.669$ & 0.021 \\
\hline$\geq 12$ & Reference & & & Reference & & \\
\hline \multicolumn{7}{|c|}{ Number of metastatic sites } \\
\hline$<3$ & 0.508 & $0.369-0.699$ & $<0.001$ & 0.651 & $0.497-0.854$ & 0.002 \\
\hline$\geq 3$ & Reference & & & Reference & & \\
\hline \multicolumn{7}{|l|}{ Visceral metastasis } \\
\hline No & 1.082 & $0.764-1.532$ & 0.657 & 0.880 & $0.690-1.122$ & 0.302 \\
\hline Yes & Reference & & & Reference & & \\
\hline \multicolumn{7}{|c|}{ Anthracycline and/or taxane } \\
\hline No & 0.749 & $0.547-1.026$ & 0.072 & 0.992 & $0.637-1.547$ & 0.973 \\
\hline Yes & Reference & & & Reference & & \\
\hline \multicolumn{7}{|l|}{ Menopausal status } \\
\hline Premenopausal & 1.185 & $0.854-1.645$ & 0.310 & 1.434 & $1.089-1.899$ & 0.010 \\
\hline Postmenopausal & Reference & & & Reference & & \\
\hline \multicolumn{7}{|c|}{ PBCT used as first-line chemotherapy } \\
\hline No & 1.532 & $1.142-2.056$ & 0.004 & 1.391 & $1.092-1.773$ & 0.008 \\
\hline Yes & Reference & & & Reference & & \\
\hline \multicolumn{7}{|c|}{ Objective response status } \\
\hline OR & 0.438 & $0.324-0.592$ & $<0.001$ & 0.448 & $0.353-0.568$ & $<0.001$ \\
\hline Non-OR & Reference & & & Reference & & \\
\hline \multicolumn{7}{|c|}{ Cox regression results of OS } \\
\hline \multirow{2}{*}{ Variables } & \multicolumn{3}{|c|}{ Training $(\mathrm{n}=236)$} & & on $(n=360)$ & \\
\hline & Hazard ratio & $95 \% \mathrm{CI}$ & p-value & Hazard ratio & $95 \% \mathrm{CI}$ & p-value \\
\hline Age, years & & & & & & \\
\hline$<40$ & 0.718 & $0.455-1.132$ & 0.154 & 0.864 & $0.618-1.207$ & 0.390 \\
\hline$\geq 40$ & Reference & & & Reference & & \\
\hline ECOG & & & & & & \\
\hline 0 & 0.695 & $0.501-0.964$ & 0.029 & 0.931 & $0.707-1.226$ & 0.612 \\
\hline$\geq 1$ & Reference & & & Reference & & \\
\hline DFI & & & & & & \\
\hline$<12$ & 2.126 & $1.445-3.128$ & $<0.001$ & 1.888 & $1.477-2.413$ & $<0.001$ \\
\hline$\geq 12$ & Reference & & & Reference & & \\
\hline Number of metastati & & & & & & \\
\hline$<3$ & 0.439 & $0.311-0.620$ & $<0.001$ & 0.497 & $0.376-0.656$ & $<0.001$ \\
\hline$\geq 3$ & Reference & & & Reference & & \\
\hline Visceral metastasis & & & & & & \\
\hline No & 0.962 & $0.666-1.390$ & 0.836 & 0.907 & $0.703-1.169$ & 0.449 \\
\hline Yes & Reference & & & Reference & & \\
\hline Anthracycline and/o & & & & & & \\
\hline No & 0.684 & $0.495-0.946$ & 0.022 & 0.581 & $0.360-0.939$ & 0.027 \\
\hline Yes & Reference & & & Reference & & \\
\hline Menopausal status & & & & & & \\
\hline Premenopausal & 1.123 & $0.794-1.588$ & 0.510 & 1.305 & $0.99-1.726$ & 0.058 \\
\hline Postmenopausal & Reference & & & Reference & & \\
\hline PBCT used as first-li & & & & & & \\
\hline No & 0.968 & $0.710-1.320$ & 0.839 & 0.816 & $0.637-1.046$ & 0.108 \\
\hline Yes & Reference & & & Reference & & \\
\hline Objective response s & & & & & & \\
\hline OR & 0.602 & $0.440-0.824$ & 0.002 & 0.561 & $0.440-0.715$ & $<0.001$ \\
\hline Non-OR & Reference & & & Reference & & \\
\hline
\end{tabular}

Abbreviations: DFI - disease-free interval; ECOG - Eastern Cooperative Oncology Group; HR - hazard ratio; MBC - metastatic breast cancer; OR - objective response; OS - overall survival; PBCT - platinum-based chemotherapy; PFS - progression-free survival 
tial single-agent chemotherapy because of its high response rate [18], but it also produced more toxicity. In the metastatic breast cancer settings, the achievement of an OR to chemotherapy was associated with a true survival benefit $[19,20]$. However, the results differed [21] and made the clinical practice difficult. No clinical trial or literature to date has demonstrated that the OR of first-line chemotherapy in mTNBC could predict the OS. The results of the present study showed that the OR was an independent prognostic factor for not only PFS but also OS. Although the first-line chemotherapy differed, the OR was still associated with greatly improved survival, confirming the validity of the OR to different treatment modalities. The OS surprisingly improved with almost 8-10 months in both the training cohort (OR vs non-OR, 23.72 vs 13.83 months, HR, 0.637, $\mathrm{p}=0.002$ ) and the validation cohort (OR vs non-OR, 21.95 vs 13.80 months, $\mathrm{HR}, 0.608, \mathrm{p}<0.001)$. This improvement indicated that TNBC, not like other subtypes of breast cancer, might be in need of combination chemotherapy or intense chemotherapy to achieve a high response rate for survival.

TNBC may harbor a dysfunctional BRCA pathway and thus may be sensitive to agents such as platinum that selectively target cells deficient in homologous recombination DNA repair $[22,23]$. The present study showed that PBCT used as first-line chemotherapy was an independent prognostic factor for PFS, but not for OS, which was in accordance with the data from the prospective phase III trial CBCSG006 [6] and the retrospective study by Zhang et al. in the Center [24]. TNBC is a heterogeneous disease divided into six intrinsic molecular subtypes: two basal-like subtypes (BL1 and BL2) sensitive to cisplatin, an immunomodulatory (IM) subtype, a mesenchymal (M) subtype, a mesenchymal stem-like subtype, and a luminal androgen receptor subtype [25]. The unselected mTNBC treated with platinum may lead to clinical heterogeneity. Furthermore, the second-line or subsequent line treatment in the present study differed, and some patients might have crossed over to platinum-based chemotherapy when the disease progressed in first-line chemotherapy. All these reasons might have narrowed down the differences in OS between the two groups.

This study had limitations due to its retrospective nature and treatment selection biases as follows. The validation cohort included only one institution, and some of the baseline information in the two cohorts was different. The comparison of survival by OR might also be biased because patients needed to live long enough to see a response to the treatment. Patients who died earlier before they had had a chance to respond to the treatment might be classified as non-responders and had an impact upon the survival estimates. A long-term observation might narrow the bias gap.

In summary, the present study comprehensively investigated the predictive value of OR in the first-line setting of mTNBC patients. It provided evidence that patients who achieved their best response of CR or PR in any first-line treatment would have prolonged survival. Therefore, it was assumed that in clinical practice, maximum tumor shrinkage and combination chemotherapy or intense chemotherapy in the first-line treatment should be considered for this aggressive subtype of breast cancer, which might help improve patients' prognosis and prolong their survival.

Acknowledgments: The authors thank the patients who shared their experiences with the medical oncology physicians, as well as the network of investigators, research nurses, study coordinators, and operation staff. In particular, they would like to thank Yunhua $\mathrm{Lu}$ for helping in data collection and Miao Mo for statistical advice.

\section{References}

[1] FOULKES WD, SMITH IE, REIS-FILHO JS. Triple-negative breast cancer. N Engl J Med 2010; 363: 1938-1948. https://doi.org/10.1056/NEJMra1001389

[2] ROBSON M, IM SA, SENKUS E, XU BH, DOMCHEK SM et al. Olaparib for Metastatic Breast Cancer in Patients with a Germline BRCA Mutation. N Engl J Med 2017; 377: 523533. https://doi.org/10.1056/NEJMoa1706450

[3] LIEDTKE C, MAZOUNI C, HESS KR, ANDRE F, TORDAI A et al. Response to neoadjuvant therapy and longterm survival in patients with triple-negative breast cancer. J Clin Oncol 2008; 26: 1275-1281. https://doi.org/10.1200/ JCO.2007.14.4147

[4] GREENBERG PA, HORTOBAGYI GN, SMITH TL, ZIEGLER LD, FRYE DK et al. Long-term follow-up of patients with complete remission following combination chemotherapy for metastatic breast cancer. J Clin Oncol 1996; 14: 2197-2205. https://doi.org/10.1200/JCO.1996.14.8.2197

[5] GENNARI A, STOCKLER M, PUNTONI M, SORMANI M, NANNI $O$ et al. Duration of chemotherapy for metastatic breast cancer: a systematic review and meta-analysis of randomized clinical trials. J Clin Oncol 2011; 29: 2144-2149. https://doi.org/10.1200/JCO.2010.31.5374

[6] HU XC, ZHANG J, XU BH, CAI L, RAGAZ J et al. Cisplatin plus gemcitabine versus paclitaxel plus gemcitabine as first-line therapy for metastatic triple-negative breast cancer (CBCSG006): a randomised, open-label, multicentre, phase 3 trial. Lancet Oncol 2015; 16: 436-446. https://doi. org/10.1016/S1470-2045(15)70064-1

[7] HAMMOND ME, HAYES DF, DOWSETT M, ALLRED DC, HAGERTY KL et al. American Society of Clinical Oncology/ College Of American Pathologists guideline recommendations for immunohistochemical testing of estrogen and progesterone receptors in breast cancer. J Clin Oncol 2010; 28: 2784-2795. https://doi.org/10.1200/JCO.2009.25.6529

[8] WOLFF AC, HAMMOND ME, HICKS DG, DOWSETT M, MCSHANE LM et al. Recommendations for human epidermal growth factor receptor 2 testing in breast cancer: American Society of Clinical Oncology/College of American Pathologists clinical practice guideline update. J Clin Oncol 2013; 31: 3997-4013. https://doi.org/10.1200/ JCO.2013.50.9984 
[9] EISENHAUER EA, THERASSE P, BOGAERTS J SCHWARTZ LH, SARGENTE D et al. New response evaluation criteria in solid tumors: revised RECIST guideline (version 1.1). Eur J Cancer 2009; 45: 228-247. https://doi. org/10.1016/j.ejca.2008.10.026

[10] DENT R, TRUDEAU M, PRITCHARD KI, HANNA WM, KAHN HK et al. Triple-negative breast cancer: clinical features and patterns of recurrence. Clin Cancer Res 2007; 13: 4429-4434. https://doi.org/10.1158/1078-0432.CCR-063045

[11] KASSAM F, ENRIGHT K, DENT R, DRANITSARIS G, MYERS $J$ et al. Survival outcomes for patients with metastatic triple-negative breast cancer: implications for clinical practice and trial design Clin Breast Cancer 2009; 9: 29-33. https://doi.org/10.3816/CBC.2009.n.005

[12] VON MINCKWITZ G, UNTCH M, BLOHMER JU, EIDTMANN H, FASCHING PA et al. Definition and impact of pathologic complete response on prognosis after neoadjuvant chemotherapy in various intrinsic breast cancer subtypes. J Clin Oncol 2012; 30: 1796-1804. https://doi. org/10.1200/JCO.2011.38.8595

[13] SIKOV WM, BERRY DA, PEROU CM, SINGH B, CIRRINCIONE CT et al. Impact of the addition of carboplatin and/or bevacizumab to neoadjuvant once-per-week paclitaxel followed by dose-dense doxorubicin and cyclophosphamide on pathologic complete response rates in stage II to III triple-negative breast cancer: CALGB 40603 (Alliance). J Clin Oncol 2015; 33: 13-21. https://doi.org/10.1200/ JCO.2014.57.0572

[14] VON MINCKWITZ G, SCHNEEWEISS A, LOIBL S, SALAT C, DENKERT C et al. Neoadjuvant carboplatin in patients with triple-ne gative and HER2-positive early breast cancer (GeparSixto; GBG 66): a randomised phase 2 trial. Lancet Oncol 2014; 15: 747-756. https://doi.org/10.1016/ S1470-2045(14)70160-3

[15] BERRUTI A, AMOROSO V, GALLO F, BERTAGLIA V, SIMONCINI E et al. Pathologic complete response as a potential surrogate for the clinical outcome in patients with breast cancer after neoadjuvant therapy: a meta-regression of 29 randomized prospective studies. J Clin Oncol 2014; 32: 3883-3891. https://doi.org/10.1200/JCO.2014.55.2836

[16] CORTAZAR P, ZHANG L, UNTCH M, MEHTA K, COSTANTINO JP et al. Pathological complete response and long-term clinical benefit in breast cancer: the CTNeoBC pooled analysis. Lancet 2014; 384: 164-172. https://doi. org/10.1016/S0140-6736(13)62422-8
[17] VON MINCKWITZ G, LOIBL S, SCHNEEWEISS A, SALAT $\mathrm{C}, \mathrm{REZAI} \mathrm{M}$ et al. Early survival analysis of the randomized phase II trial investigating the addition of carboplatin to neoadjuvant therapy for triple-negative and HER2-positive early breast cancer (GeparSixto). Cancer Res 2016; 76: 8-12. https://doi.org/10.1158/1538-7445.SABCS15-S2-04

[18] CARRICK S, PARKER S, THORNTON CE, GHERSI D, SIMES J et al. Single agent versus combination chemotherapy for metastatic breast cancer. Cochrane Database Syst Rev 2009; 2009: CD003372. https://doi.org/10.1002/14651858. CD003372.pub3

[19] BRUZZI P, DEL MASTRO L, SORMANI MP, BASTHOLT L, DANOVA $\mathrm{M}$ et al. Objective response to chemotherapy as a potential surrogate end point of survival in metastatic breast cancer patients. J Clin Oncol 2005; 23: 5117-5125. https:// doi.org/10.1200/JCO.2005.02.106

[20] PIERGA JY, ROBAIN M, JOUVE M, ASSELAIN B, DIERAS $\mathrm{V}$ et al. Response to chemotherapy is a major parameter-influencing long-term survival of metastatic breast cancer patients. Ann Oncol 2001; 12: 231-237. https://doi. org/10.1023/a:1008330527188

[21] DEAR RF, MCGEECHAN K, JENKINS MC, BARRATT A, TATTERSALL $\mathrm{MH}$ et al. Combination versus sequential single agent chemotherapy for metastatic breast cancer. Cochrane Database Syst Rev 2013; 12: CD008792. https://doi. org/10.1002/14651858.CD008792.pub2

[22] GONZALE-ANGULO AM, TIMMS KM, LIU S, CHEN H, LITTON JK et al. Incidence and outcome of BRCA mutations in unselected patients with triple receptor-negative breast cancer. Clin Cancer Res 2011; 17: 1082-1089. https:// doi.org/10.1158/1078-0432.CCR-10-2560

[23] KWON JS, GUTIERREZ-BARRARA AM, YOUNG D, SUN CC, DANIELS MS et al. Expanding the criteria for BRCA mutation testing in breast cancer survivors. J Clin Oncol 2010; 28: 4214-4220. https://doi.org/10.1200/JCO.2010.28.0719

[24] ZHANG J, FAN M, XIE J, WANG Z, WANG B et al. Chemotherapy of metastatic triple negative breast cancer: Experience of using platinum-based chemotherapy. Oncotarget 2015; 6: 43135-43143. https://doi.org/10.18632/oncotarget. 5654

[25] LEHMANN BD, BAUER JA, CHEN X, SANDERS ME, CHAKRAVARTHY AB et al. Identification of human triplenegative breast cancer subtypes and preclinical models for selection of targeted therapies. J Clin Invest 2011; 121: 27502767. https://doi.org/10.1172/JCI45014 